\title{
BMJ Open Quality Are they high on steroids? Tailored interventions help improve screening for steroid-induced hyperglycaemia in hospitalised patients
}

\author{
Punith Kempegowda, ${ }^{1,10}$ Alana C Livesey, ${ }^{2}$ Laura McFarlane-Majeed, ${ }^{3}$ \\ Joht Singh Chandan, ${ }^{2}$ Theresa Smyth, ${ }^{2}$ Martha Stewart, ${ }^{2}$ Karen Blackwood, ${ }^{2}$ \\ Michelle McMahon, ${ }^{2}$ Anitha Vijayan Melapatte, ${ }^{4}$ Sofia Salahuddin, ${ }^{2}$ \\ Jonathan Webber, ${ }^{2}$ Sandip Ghosh ${ }^{2}$
}

\begin{abstract}
To cite: Kempegowda P, Livesey AC, McFarlaneMajeed L, et al. Are they high on steroids? Tailored interventions help improve screening for steroid-induced hyperglycaemia in hospitalised patients.BMJ Open Quality 2018;7:e000238. doi:10.1136/ bmjoq-2017-000238
\end{abstract}

Received 26 0ctober 2017 Revised 28 December 2017 Accepted 24 January 2018

A Check for updates

${ }^{1}$ Department of Diabetes, Endocrinology and General Internal Medicine, Heart of England NHS Foundation Trust, Birmingham, UK

${ }^{2}$ Department of Diabetes, University Hospital Birmingham NHS Foundation Trust,

Birmingham, UK

${ }^{3}$ Medical School, University of Birmingham, Birmingham, UK

${ }^{4}$ Department of Health

Informatics, Queen Elizabeth

Hospital Birmingham,

Birmingham, UK

${ }^{10}$ Institute of Metabolism and

Systems Research, University of Birmingham, Birmingham, United Kingdom

Correspondence to Dr Punith Kempegowda; p.kempegowda@nhs.net

\section{ABSTRACT}

Steroid-induced hyperglycaemia (SIH) is a common adverse effect in patients both with and without diabetes. This project aimed to improve the screening and diagnosis of SIH by improving the knowledge of healthcare professionals who contribute to the management of SIH in hospitalised patients. Monitoring and diagnosis of $\mathrm{SIH}$ were measured in areas of high steroid use in our hospital from May 2016 to January 2017. Several interventions were implemented to improve knowledge and screening for SIH including a staff education programme for nurses, healthcare assistants and doctors. The Trust guidelines for SIH management were updated based on feedback from staff. The changes to the guideline included shortening the document from 14 to 4 pages, incorporating a flowchart summarising the management of $\mathrm{SIH}$ and publishing the guideline on the Trust intranet. A questionnaire based on the recommendations of the Joint British Diabetes Societies for SIH was used to assess the change in knowledge pre-intervention and post-intervention. Results showed an increase in junior doctors' knowledge of this topic. Although there was an initial improvement in screening for SIH, this returned to near baseline by the end of the study. This study highlights that screening for SIH can be improved by increasing the knowledge of healthcare staff. However, there is a need for ongoing interventions to sustain this change.

\section{BACKGROUND}

Steroids are used in a variety of settings including allergic, inflammatory and immunological conditions. The prevalence of steroid use in hospitalised patients is as high as $12 \%{ }^{1}$ Steroids act directly on the liver to increase gluconeogenesis, as well as increasing protein and fat breakdown. There is also a reduction in beta-cell function/insulin secretion in the pancreas. In addition, steroids simultaneously cause insulin resistance throughout the body, which further exacerbates hyperglycaemia. This combination of effects is commonly known as steroid-induced hyperglycaemia $(\mathrm{SIH})$ and is defined by the Joint
British Diabetes Society (JBDS) for inpatient management as a capillary blood glucose (CBG) $>11.1 \mathrm{mmol} / \mathrm{L}$ measured on two separate days in the pre-evening meal period. ${ }^{2}$ It is important to note that this occurs in patients both with and without diabetes. ${ }^{2}$ The prevalence of hyperglycaemia in patients treated with steroids has been previously identified between $64 \%$ and $72 \% .^{3-5}$

Hyperglycaemia for prolonged periods may cause symptoms of diabetes including polydipsia, polyuria, fatigue, infections and other complications. ${ }^{5}$ This may also manifest in diabetic emergencies such as hyperosmolar hyperglycaemic state or diabetic ketoacidosis. A guideline for monitoring and management of SIH has been published by the JBDS for inpatient management. ${ }^{2}$ Several audit standards have been set out in this document for inpatient management of SIH.

Through informal discussions on the wards and from the quality of hyperglycaemia referrals sent to diabetes team for inpatient review, we identified staff knowledge was poor surrounding the management of SIH. In addition, it was acknowledged that the trust guideline was both long (14 pages) and difficult to follow as it was made up of paragraphs of text. We hypothesised that monitoring and diagnosis of SIH could be increased through education of staff and improvement of local guideline, which formed the basis of this quality improvement project (QIP). This project involved assessing change in the screening for SIH and knowledge among healthcare practitioners with several interventions.

\section{PROBLEM}

An audit assessing the current management of SIH in our hospital showed that the prevalence of $\mathrm{SIH}$ was $25 \%$. SIH was defined as two 


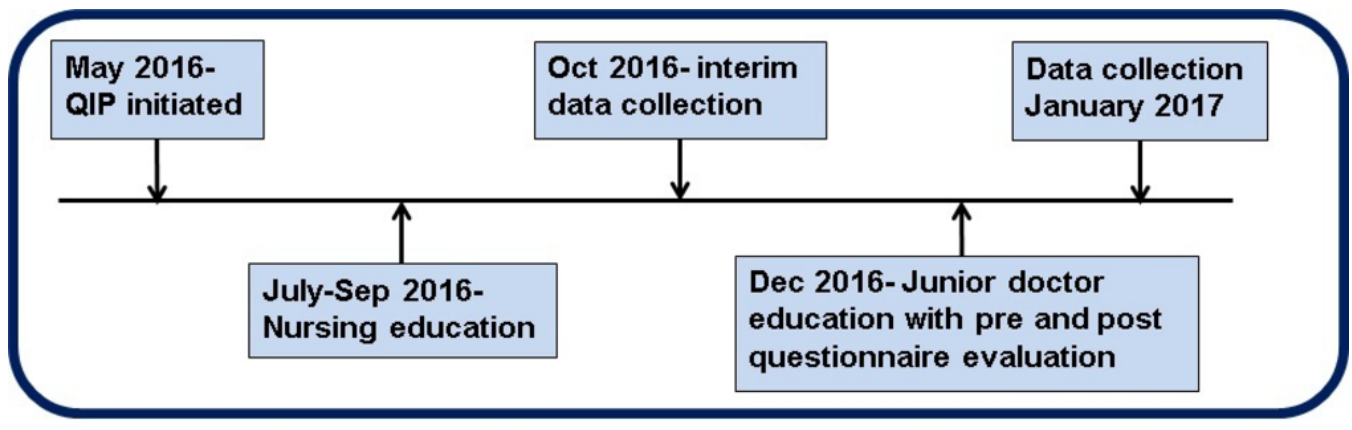

Figure 1 Quality improvement project (QIP) milestones May 2016-January 2017.

measurements of CBG greater than $11.1 \mathrm{mmol} / \mathrm{L}$ as per the JBDS-IP guideline. This prevalence is only inclusive of those patients who were appropriately screened for $\mathrm{SIH}$; of this cohort, $75 \%$ of patients had their CBG measured at least once in the pre-evening time frame. We noted that screening for SIH including pre-evening meal CBG, glycated haemoglobin (HbAlC), confirmatory serum glucose and diabetes referrals were higher in patients with diabetes than those without, but fell far lower than the national guidelines' audit recommendations of $90 \%$.

The monitoring, diagnosis and management of $\mathrm{SIH}$ involve input from different members of the medical team. Doctors participate by prescribing steroids, requesting blood tests and arranging diabetes referrals. Nurses and healthcare assistants (HCAs) are involved in CBG monitoring. There are many issues with this framework, including the requirement of good understanding of SIH by staff and communication between team members to ensure adequate monitoring is carried out and the results acted on. We found limited studies assessing the knowledge and management of SIH in the clinical setting.

\section{DESIGN}

The QIP was undertaken between May 2016 and January 2017 at a large tertiary-care centre located in the West Midlands, providing services to a diverse population of patients with over 375000 admissions per year. Six wards with high steroid use (respiratory, neurology and oncology wards) were selected to maximise the impact of the focused intervention. The standards for monitoring and diagnosis of SIH were based on JBDS-IP guideline ${ }^{2}$ We included all patients treated with two or more doses of oral/intramuscular/intravenous or subcutaneous steroids during the study period. Patients treated with inhaled steroids or 'oneoff' steroids were excluded in the current study. Baseline, interim and final data were collected in May 2016, October 2016 and January 2017. The aim was to:

1. Improve CBG screening in patients receiving steroids to $>90 \%$.

2. Improve the proportion of patients with adequate blood glucose control to $>75 \%$.

3. Improve the knowledge regarding monitoring, diagnosis and management of SIH among healthcare practitioners.
We adopted the plan-do-study-act (PDSA) cycle to address the primary outcome in this study. The PDSA cycle method is described $a^{6}$ :

1. Plan-plan the test, intervention or observation, including a plan for collecting data.

2. Do-trial the intervention on a small scale.

3. Study - analyse the data and study the results.

4. Act-refine the change, based on what was learnt from the test.

The interventions and date of their introduction into the QIP are summarised in figure 1.

\section{STRATEGY}

A working group consisting of three diabetes consultants, one diabetes registrar with a special interest in $\mathrm{SIH}$, three diabetes specialist nurses (DSNs), two junior doctors and one fourth-year medical student was set up to act as the main steering group to ensure completion of the QIP. The steering group were responsible for reviewing the literature and identifying effective strategies that would be employed in the PDSA cycles. The process was iterative as the steering group sought feedback from junior doctors and nurses regarding areas that needed specific improvement. During the study period, the steering group identified three important themes relating to improving the identification and management of SIH, which in turn formed the basis of the three PDSA cycles used in the study to achieve our aims:

1. Improvement of CBG monitoring to screen for SIH by nursing and auxiliary staff.

2. Improvement of junior doctors' knowledge regarding SIH.

3. Improvement of the Trust guideline for SIH.

PDSA cycle 1: improvement of CBG monitoring to screen for SIH by nursing and auxiliary staff

DSNs and diabetes educators conducted one-to-one training for nurses and HCAs on the sampled wards about SIH. The DSNs used a flowchart developed for the study (figure 2) and teaching was carried out between June and September 2016. One-to-one teaching is a validated teaching tool used in a wide variety of settings. ${ }^{78}$ The flowchart and tutorials included information about diagnostic criteria, the correct timing and duration of CBG monitoring, and appropriate 


\section{Steroid Induced Hyperglycaemia guidelines}

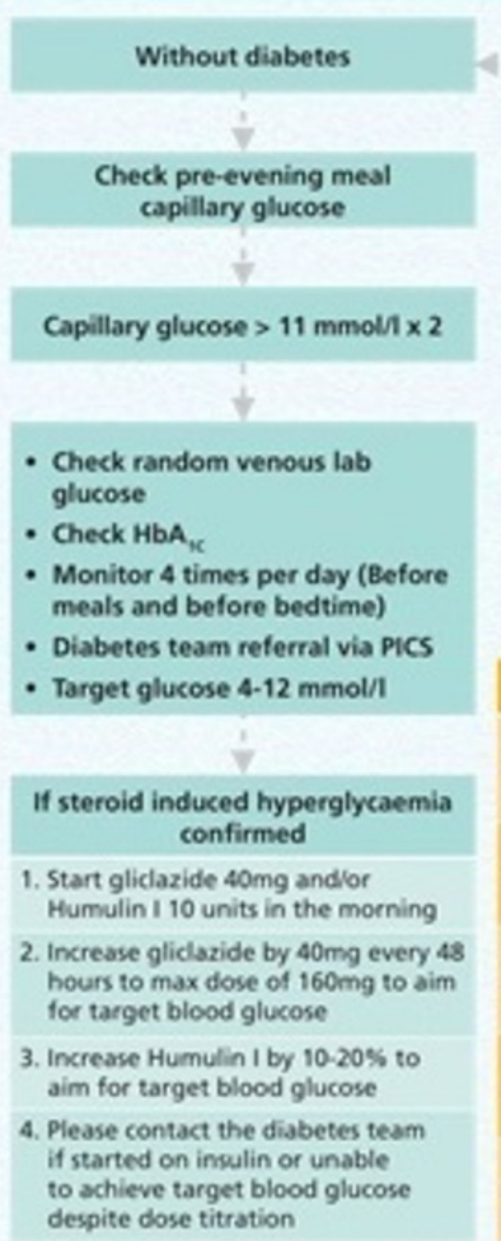

\section{Steroid \\ started}

eck pre-evening mest

nat

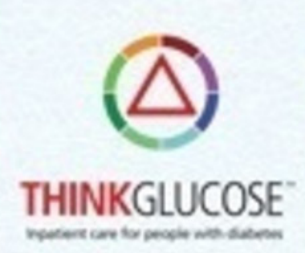

- Check HbA

- Diabetes team referral via PICS

- Target glucose 4-12 mmol/1

Monitor capillary glucose 4 times per day (Before meals and before bedtime)

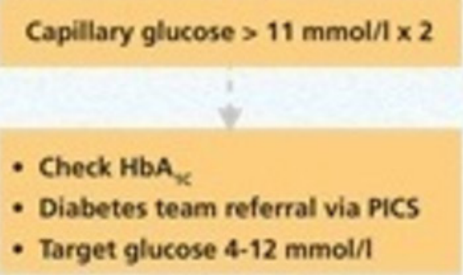

\section{Discharge planning for all patients with steroid induced hyperglycaemia}

- Ufestyle advice

- Hypoglycaemia advice

- Gluxose monitoring (late afternoonvevening)
- The discharging doctor to email:

Diabetes.Secretarieseuhb.nhs.uk to request OP

appointment by in diabetes clinic (code) XXBN)

- GP to repeat fasting gluxosel HbA, 3 months later

Figure 2 Flowchart to diagnose and manage steroid-induced hyperglycaemia. The flowchart included information about diagnostic criteria, appropriate capillary blood glucose monitoring and appropriate investigations required if the patient was diagnosed with SIH (PICS, patient information and communication system; OP, outpatient; GP, general practitioner).

investigations required if the patient was diagnosed with SIH. Forty-eight per cent $(114 / 237)$ of the nursing staff were educated first hand during this period. To further improve teaching uptake, we later adopted a chain education approach where the DSNs trained one member of ward staff, who then disseminated the knowledge further to other colleagues. This was highly effective, achieving up to $81 \%$ staff education.

PDSA cycle 2: improvement of junior doctors' knowledge regarding SIH

A 5 min focused teaching session was organised for junior doctors ranging from Foundation Year to Core Medical Training, which was delivered during their mandatory weekly teaching sessions in December 2016. The teaching was based on a presentation pre-approved by the steering group and delivered by the same team responsible for delivering the PDSA cycle 1 teaching. The focused teaching session included slides exploring the pathophysiology, diagnosis and management of SIH. This was facilitated using the flowchart from PDSA cycle 1 and exploration of tools to aid management on the Trust intranet. Prior to the delivery of the session, we delivered a questionnaire to measure the baseline knowledge, and this was then repeated 6 weeks following the teaching to assess for improvement in knowledge regarding SIH. 
PDSA cycle 3: improvement of the Trust guideline for SIH

Feedback from the healthcare staff suggested the Trust's current SIH guideline was too lengthy and difficult to use. During the study period, the steering group revised the current 14-page document to 4 pages, with the inclusion of the easy-to-interpret flowchart used in PDSA cycles 1 and 2. Revision and improvement of an existing guideline has been previously proven to be effective in a similar QIP conducted at the trust where the diabetes ketoacidosis treatment guideline was summarised in a single poster. This modification resulted in a vast reduction in the duration of diabetic ketoacidosis (DKA) in inpatients at the trust. ${ }^{9}$ All healthcare staff were informed of the updated version of the SIH guideline, which was published on the Trust intranet.

The steering group met up at regular intervals to discuss feedback from healthcare staff and the progress of the different cycles. Complementary to the study, the importance of SIH was highlighted to the hospital staff during the diabetes awareness week in November 2016.

\section{MEASUREMENT AND ANALYSIS}

Staff practice was assessed at three points: (1) baseline in May 2016, (2) post-nursing and HCA training (PDSA 1) in October 2016, and (3) post-junior doctor training and guideline updates (PDSA 2 and 3) in January 2017. We included patients prescribed steroids on the selected wards during these 3 months. Demographic data, medical history of diabetes, steroid type, prescription duration and presence of pre-evening meal glucose measurement were collected for patients admitted on the study wards. Steroid dose was calculated in hydrocortisone equivalent units to allow direct comparison of doses. ${ }^{10}$ Once an individual fulfilled the diagnostic criteria for SIH (figure 2), further glucose measurements in addition to pre-evening CBGs were also analysed to assess if patients were appropriately having their blood glucose monitored four times per day. Junior doctors were assessed on their SIH knowledge using a nine-question survey (Appendix xx) pre-intervention and 6weeks' post-educational intervention as outlined above in PDSA cycle 2. The questionnaire was approved by the steering group and trialled in a pilot study of 28 members to ensure its appropriateness. Statistical analysis was performed using the two-proportion $\mathrm{Z}$ test where $\mathrm{H}_{0}: \mathrm{P}_{1}=\mathrm{P}_{2}$. P (hat) values were calculated for each set of data and then used to calculate $\mathrm{Z}$ values.

\section{RESULTS}

The demographics of patients included in the QIP along with the average hydrocortisone equivalent steroid doses at the three time points are summarised in table 1 . There were 68 patients treated with steroids on the six wards in May 2016. Also, 19.1\% (13/68) had adequate pre-evening CBG measurement, with $25.0 \%(17 / 68)$ of initial measurements meeting diagnostic criteria for SIH.

There was an improvement in the proportion of patients who had pre-evening meal CBG monitored at least once during their steroid therapy from May 2016 to October 2016 in both patients with and without diabetes (figure 3). This improvement corresponded to the period of nursing and HCA education. However, the change was not statistically significant. While the improvement was sustained in patients with diabetes, these results dropped in patients without diabetes in our repeat audit in January 2017, suggesting a need for sustained intervention. We noted mixed results when we measured the proportion of patients who had appropriate monitoring throughout their steroid therapy (figure 4). While there was an improvement from May 2016 to October 2016 for patients without diabetes, the values returned to baseline in our repeat audit in January 2017. Interestingly, for patients with diabetes, there was a drop in comparative values from May 2016 to October 2016, whereas the pre-evening CBG monitoring improved during this period in this subgroup. The cause of the reduced overall monitoring in this period was not identified during this QIP. However, these values improved in our repeat audit in January 2017.

The changes in screening were reflected in the diagnosis of SIH during our study (figure 5). One hundred per cent $(15 / 15)$ of patients with diabetes had SIH in our interim analysis in October 2016 compared with $50 \%$ $(13 / 26)$ in May 2016. However, this dropped to $56.3 \%$ $(9 / 16)$ in January 2017. Of our collected measurements, $12.7 \%(8 / 63)$ of patients without diabetes had SIH in October 2016 compared with 9.5\% (4/42) in May 2016. This dropped to $4.8 \%(3 / 63)$ in January 2017.

\begin{tabular}{llll}
\hline \multicolumn{4}{l}{ Table 1 Sociodemographics of patients included in the quality improvement project } \\
\hline Characteristics & May 2016 & October 2016 & January 2017 \\
\hline Mean age (years) & $62 \pm 17.1$ & $60 \pm 17.7$ & $62 \pm 16.5$ \\
Age range (years) & $20-95$ & $19-91$ & $18-96$ \\
Male:female ratio & $1.5: 1$ & $1.3: 1$ & $1: 1$ \\
Dose (hydrocortisone equivalent dose/24hours) & 1558 & 1146 & 948 \\
Average duration of steroid treatment (days) & 3.79 & 3.95 & 5.4 \\
\hline
\end{tabular}

There was a significant improvement in junior doctors' knowledge regarding route of steroids causing steroid-induced hyperglycaemia, monitoring and follow-up required on discharge. 

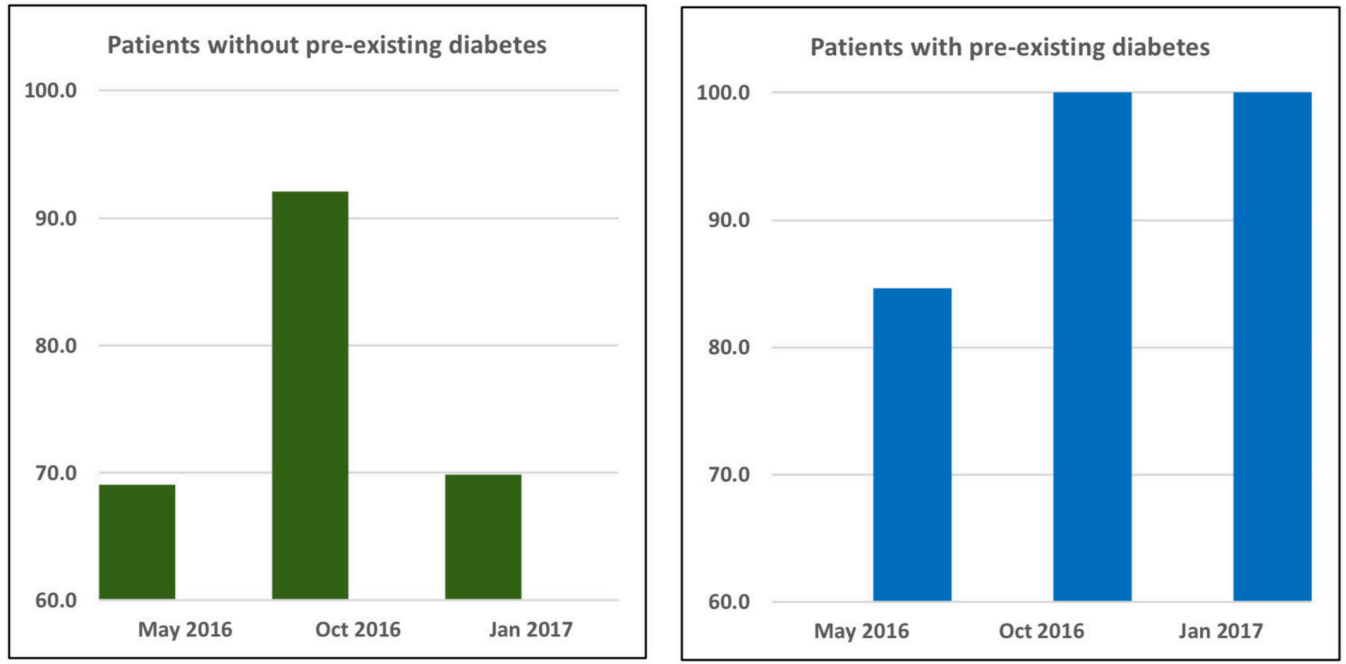

Figure 3 Percentage of patients who had their pre-evening capillary blood glucose monitored at least once during their steroid therapy. There was an improvement in the monitoring from May 2016 to October 2016 in both patients with and without diabetes. While the improvement sustained in patients with diabetes, similar results dropped in patients without diabetes in our repeat audit in January 2017, suggesting a need for sustained intervention.

Seventy-eight doctors completed pre-teaching questionnaire and observed the teaching presentation, and 58 completed the follow-up questionnaire. There was an equal distribution of the different grades of doctors' responding in the pre-questionnaire and post-questionnaire. There was a significant improvement in junior doctors' knowledge regarding the route of steroids causing SIH $(\mathrm{P}=0.01)$, screening for $\mathrm{SIH}(\mathrm{P}=0.01)$ and follow-up required on discharge if diagnosed with SIH $(\mathrm{P}=0.01)$ in the survey (table 2$)$.

\section{LESSONS AND LIMITATIONS}

While each intervention appeared to improve aspects of SIH screening, diagnosis and management, it was a combination of all of the interventions that resulted in an overall improvement. This reiterates the importance of designing interventions to include all members of healthcare staff who are involved in the management of the highlighted topic. Doctors and nurses form two of the busiest groups of healthcare staff and are diversely distributed in time (due to shift patterns) and space (across the hospital). Furthermore, there are many other important roles to perform and targets to achieve in their jobs while catering to the needs of our index patients. Therefore, it is important to design interventions that are directed to reach the biggest possible group of each job role and yet simple enough to ensure the information is retained.

One of the earliest strategies and strengths of our QIP was to involve all the stakeholders and ensure they were represented in the steering group. The group represented diabetes consultants, DSNs, diabetes educators, junior doctors and medical students with special interest
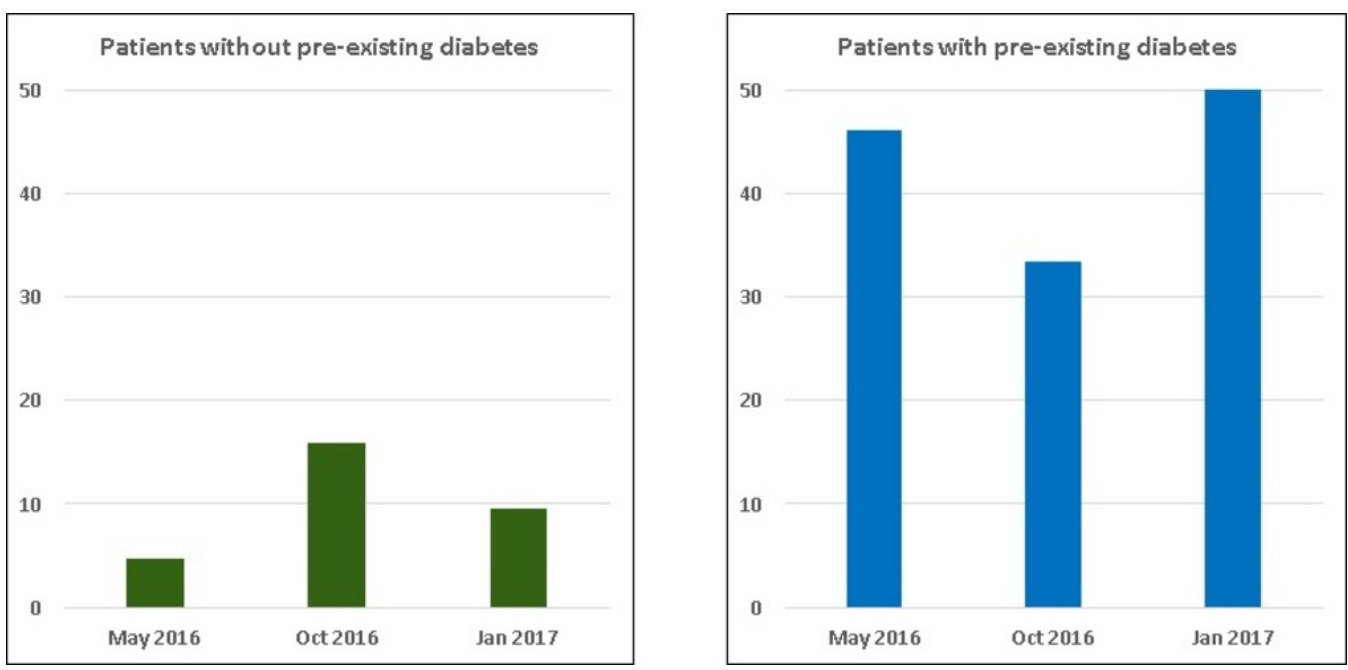

Figure 4 Percentage of patients with appropriate capillary blood glucose monitoring as per guidelines while on steroids. While patients without diabetes had better monitoring in our interim analysis, this returned to near baseline in repeat measures. There was a drop in adequate monitoring in patients with diabetes during interim analysis, which improved at the end of the study. 

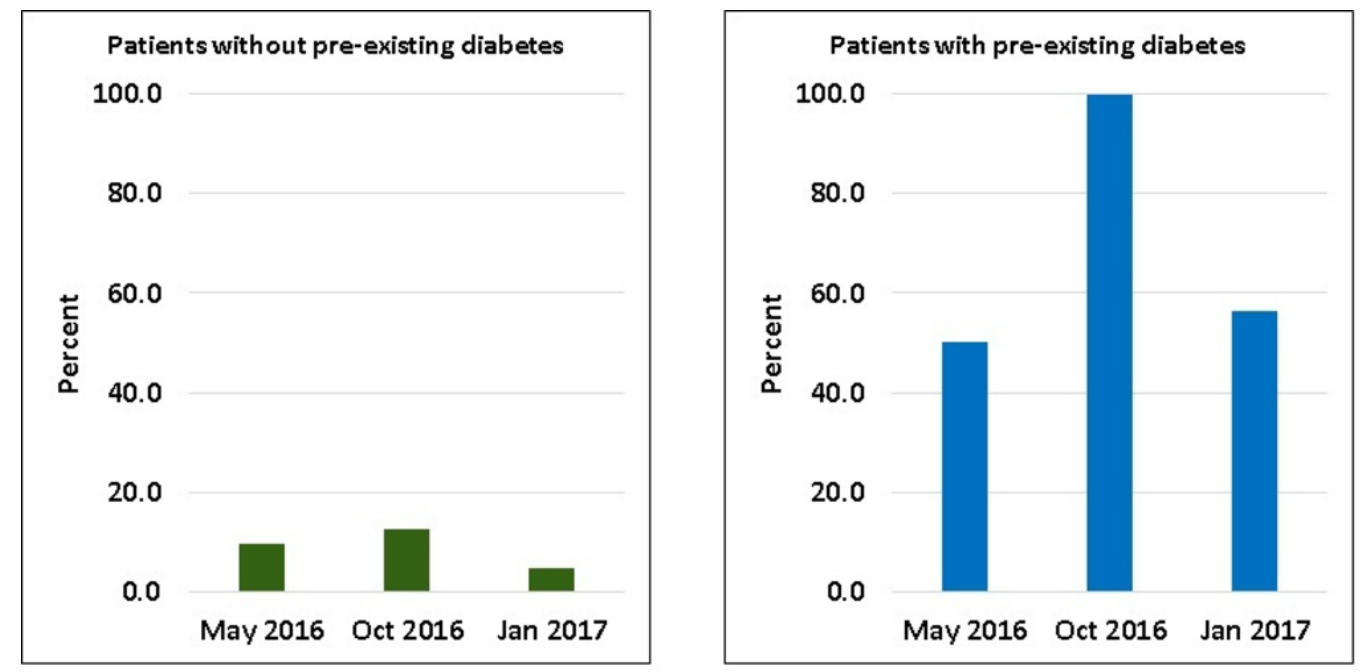

Figure 5 Percentage of patients who developed steroid-induced hyperglycaemia (SIH) during the study. There was an improvement in the diagnosis of SIH in both patients with and without diabetes in October 2017. However, this dropped back to baseline in repeat audit in January 2017.

in SIH. A multidisciplinary steering group allowed for critical analysis of each intervention and expertise to ensure interventional appropriateness to each staff grade level. This approach would be applicable to other educational QIPs.

Decentralising the educational interventions appeared as a strength in our QIP; this could be applied in other settings for successful educational interventions. PDSA cycle 1 used DSNs trained in SIH management who reached out to nurses and HCAs on target wards. This created an interactive learning environment and helped the steering group obtain feedback about the practical difficulties faced by staff managing SIH. The feedback, alongside input from junior doctors, helped us redesign the Trust's management protocol (PDSA cycle 3). Comparatively for the junior doctors, due to fluidity in their rotas in both time and location, the steering group felt this approach would not be appropriate for this group. Instead, we used a previously trialled and successful method in the trust of delivering short educational interventions during centralised teaching. The impact of this was reflected in the improvement of junior doctors' knowledge regarding SIH. This result reiterates the usefulness of this setting for the delivery of short educational interventions.

Despite the initial success, we noted a drop in glucose monitoring in our index wards that we postulated as an effect of high staff turnover, which dilutes the effect of education. We noted a similar result in another QIP to improve DKA management at our Trust. However, in the DKA project, there was a significant improvement over the longer term when all interventions were in place. We are currently continuing regular education about SIH for nurses and HCAs through a 'back to the floor' programme. This programme involves DSNs returning to wards to reinforce teaching and aims to maintain the

\begin{tabular}{llll}
\hline \multicolumn{2}{l}{ Table 2 Junior doctors' questionnaire responses pre-teaching and post-teaching } & & \\
\hline Question & Pre & Post & P value \\
\hline Which patients are at risk of SIH? & 76.92 & 80.39 & NA \\
Which routes of steroids can cause SIH? & 46.15 & 74.51 & 0.01 \\
What is the diagnostic CBG for SIH? & 74.36 & 74.51 & NA \\
$\begin{array}{l}\text { What monitoring should be carried out when steroids are } \\
\text { prescribed? }\end{array}$ & 57.69 & 64.71 & NA \\
What time should the diagnostic CBG be done? & 32.05 & 64.71 & 0.01 \\
What monitoring should occur once diagnosed with SIH? & 44.87 & 68.63 & 0.01 \\
What further action should be taken if diagnosed with SIH? & 64.10 & 76.47 & NA \\
What monitoring should occur when steroids are stopped? & 78.21 & 82.35 & NA \\
What discharge arrangements should be made if patient had SIH? 44.87 & 70.59 & 0.01 \\
\hline Total score & 57.69 & 72.98 & NA \\
\hline
\end{tabular}

There was a significant improvement in junior doctors' knowledge regarding route of steroids causing SIH, monitoring and follow-up required on discharge.

CBG, capillary blood glucose; NA, not applicable; SIH, steroid-induced hyperglycaemia. 
positive changes made through education in these projects. Early indications show this to be an effective way of improving several indicators of diabetes care, which would be transferable to screening for SIH.

Although the QIP was largely successful, there were important limitations which other QIPs or audits could learn from. This QIP was limited to hospitalised patients and thus not directed towards the biggest group of patients using steroids-those managed in outpatient clinics; therefore, future work could be done to assess SIH monitoring and management in these individuals. Our current QIP was limited to wards with high steroid use, which may imply a systematic bias as the doctors and nursing staff may experience SIH more frequently in these settings; therefore, their baseline understanding and experience in managing these patients may be better.

As the work was split into three PDSA cycles, data collection was also staggered at three separate points. Continuous data collection would have allowed for a more fluid analysis, which could have assessed the impact of each cycle further. With improvement of technology, continuous data collection is becoming easier and future iterations of this QIP at the trust will look to incorporate this. Future work will require the need to trial this style of QIP in other settings.

Although multiple indicators were recorded about the patients, absolute blood glucose level recording pre-intervention and post-intervention was not included in the study. Due to the fluctuant nature of individual readings of blood glucose levels, a much more detailed data collection process exploring other confounders including medical history and environmental factors would need to be undertaken, which was not available in this QIP due to limitations on time, resources and expertise of individuals conducting the data collection. Future work could include assessing the impact of these interventions on this important clinical outcome. An important limitation relating to the results is the accuracy of our definition of incidence of new cases of SIH. We are likely to be underestimating our true incidence of SIH due to the low numbers of adequate pre-evening CBG testing for initial screening $(19.1 \%)$. The same limitation relates to the subgroup analysis of identifying the number of patients diagnosed with SIH when comparing the patients with and without diabetes as not all cases were not appropriately screened. Future work could look at reducing this bias by only conducting subgroup analysis on those who have been appropriately screened.

Due to a low sample size, the results are not necessarily generalisable. However, the results still demonstrate the importance of a combination of interventions in improving SIH management. Due to limitations in time and resources of the individuals conducting data collection, the current QIP focused on areas of those at highest risk of developing SIH. Our future work will look to replicate this model in a larger setting with a larger population so that there would be scope to improve the breadth of the data collected and to allow for useful subgroup analysis, an example being the inclusion of the indication for the steroid by the prescriber. Due to the anticipated low sample size, these data were not recorded in this setting as analysis by indication would not have provided enough outcomes to produce meaningful results. Alongside this, baseline glycaemic control would prove to be a useful comparative characteristic to identify and account for individuals who were already more prone to developing SIH.

While the variety in grade and experience of the steering group proved to be beneficial for the study, patient input as stake holders would further strengthen the design of the QIP. Future work could focus on educating patients to know that they should have their CBG monitored, in turn becoming integral to their own care while as an inpatient and also, if required, once discharged. Although a problem with the monitoring and management of SIH has been identified in this study, the cause of this has not been isolated, nor has it in the literature. In this QIP, we focused on interventions for doctors, nurses and auxiliary staff; however, there are other multidisciplinary team members such as pharmacists and therapists who play a key role in management of such patients. Interventions targeting these staff groups could also prove beneficial.

In order to complement and sustain the educational interventions we introduced, there are other aspects of the management of SIH that could be targeted which we did not explore. At this trust, an electronic prescribing system is used through which an alert system could be initiated for patients prescribed steroids. This could prompt nursing staff to perform CBG monitoring and for doctors to check these values each day and arrange further diagnostic tests and hyperglycaemic control as required. Unfortunately, this is a system which takes up to 2 years to initiate and so could not be included in our QIP.

\section{CONCLUSION}

Tailored interventions aimed at doctors, nurses and HCAs can improve screening for SIH in hospitalised patients. While nursing education resulted in increased CBG monitoring, ongoing interventions may be needed to sustain this improvement. Short and focused educational intervention resulted in good improvement and retention of knowledge regarding SIH among junior doctors. Follow-up studies of the long-term impact on clinical practice in this area will guide further intervention.

Acknowledgements We would like to thank the diabetes specialist nurses at Queen Elizabeth Hospital Birmingham for their help and support in training the junior doctors and nurses regarding SIH. We thank Ms. Katie Bray and Ms. Denise Burton at our postgraduate education department for their help in organising training sessions for junior doctors. Last, but not the least, we thank the nursing staff and junior doctors at our Trust for their involvement in improving the standard of care for $\mathrm{SIH}$ management.

Contributors PK and ACL conceptualised the study and are joint first authors for the study. LM-M helped with study designing and data collection. JSC helped in analysing the data and revising the manuscript. AVM assisted in data collection and data mining. TS, MS, KB and MM delivered training as part of interventions to improve service. SS, JW and SG supervised the study. 
Competing interests None declared.

Provenance and peer review Not commissioned; externally peer reviewed.

Data sharing statement There are no additional unpublished data from the study.

Open Access This is an Open Access article distributed in accordance with the Creative Commons Attribution Non Commercial (CC BY-NC 4.0) license, which permits others to distribute, remix, adapt, build upon this work non-commercially, and license their derivative works on different terms, provided the original work is properly cited and the use is non-commercial. See: http://creativecommons.org/ licenses/by-nc/4.0/

(c) Published by the BMJ Publishing Group Limited. For permission to use (where not already granted under a licence) please go to http://www.bmj.com/company/ products-services/rights-and-licensing/

\section{REFERENCES}

1. Narwani V, Swafe L, Stavraka C, et al. How frequently are bedside glucose levels measured in hospital inpatients on glucocorticoid treatment? Clin Med 2014;14:327-8.

2. Diabetes UK. Management of hyperglycaemia and steroid (glucocorticoid) therapy. 2014 https://www.diabetes.org.uk/ Documents/About Us/What we say/JBDS management of hyperglycaemia and steriod therapy.pdf.
3. Tamez-Pérez HE, Quintanilla-Flores DL, Rodríguez-Gutiérrez R, et al. Steroid hyperglycemia: prevalence, early detection and therapeutic recommendations: a narrative review. World J Diabetes 2015:6:1073-81.

4. Fong AC, Cheung NW. The high incidence of steroidinduced hyperglycaemia in hospital. Diabetes Res Clin Pract 2013;99:277-80.

5. Donihi AC, Raval D, Saul M, et al. Prevalence and predictors of corticosteroid-related hyperglycemia in hospitalized patients. Endocr Pract 2006;12:358-62.

6. Gillam S, Siriwardena AN. Frameworks for improvement: clinical audit, the plan-do-study-act cycle and significant event audit. Qual Prim Care 2013;21:123-30.

7. Hawthorne K, Tomlinson S. One-to-one teaching with picturesflashcard health education for British Asians with diabetes. Br J Gen Pract 1997:47:301-4.

8. Banerjee M, Macdougall M, Lakhdar AF. Impact of a single one-toone education session on glycemic control in patients with diabetes. J Diabetes 2012;4:186-90

9. Kempegowda P, Coombs B, Nightingale P, et al. Regular and frequent feedback of specific clinical criteria delivers a sustained improvement in the management of diabetic ketoacidosis. Clin Med 2017;17:389-94.

10. Joint Formulary Committee. Glucocorticoid therapy: BNF. In: British National Formulary (online). London: BMJ Group and Pharmaceutical Press, 2017. 